\title{
Genetics of aspirin induced asthma
}

\author{
Marek Sanak, Andrzej Szczeklik
}

Aspirin induced asthma (AIA) is more common in women than in men. The first symptoms of the disease are often a viral respiratory infection which is followed by a prolonged and perennial rhinitis. ${ }^{1}$ Nasal congestion, anosmia, and rhinorrhoea are accompanied by nasal polyps which are found on physical examination in about half the patients. Within a few years bronchial asthma develops, characterised by aspirin intolerance. Aspirin precipitates life threatening attacks of asthma accompanied by rhinorrhoea, conjunctive congestion, and facial and neck flushing. The trait of aspirin sensitivity, despite avoidance of nonsteroidal anti-inflammatory drugs to which patients usually have cross sensitivity, frequently remains for the patient's lifetime.

Familial history of aspirin intolerance Although asthma, nasal polyps, and aspirin intolerance are considered as an inherited disease in the catalogue of Mendelian Inheritance in $M a n,{ }^{2}$ reports on familial occurrence of AIA are rather scarce. Miller described a pair of sisters with the trait. ${ }^{3}$ In one of the families described by Lockey et $a l^{4}$ consanguinity of the parents suggested a recessive inheritance pattern. In the other family a late onset of AIA and discordance between identical twins raised the possibility of an interaction between environmental and genetic factors. Another multiple family with mild asthma sensitive aspirin and dominant inheritance of the trait was described by von Maur et al. ${ }^{5}$ An early onset of asthma in most affected members of the family and a lack of symptoms of sinusitis or nasal polyposis distinguished this familial variant of AIA.

In almost 400 patients studied in the European Network on Aspirin Induced Asthma ${ }^{6}$ familial occurrence of aspirin hypersensitivity was reported in $5.1 \%$. In families with multiple cases of AIA, affected individuals were usually siblings. The course of their disease was characterised by an intense rhinitis and was more severe, as scored by the number of admissions to hospital, than in singleton families. We recently consulted two sisters aged 27 and 20 years with aspirin intolerance who had reported rhinitis since the age of 12 years. In the older sister aspirin challenge precipitated an asthma attack. The younger sister was suffering from perennial rhinitis and had blood eosinophilia. Following aspirin challenge she developed marked nasal symptoms leading to a profound decrease in nasal airflow and ocular irritation, but without bronchial obstruction. Both sisters shared the variant allele of leukotriene $\mathrm{C}_{4}\left(\mathrm{LTC}_{4}\right)$ synthase associated with AIA. Although their parents and some other family members were atopic or had moderate eosinophilia, the aspirin challenge was negative in all.

\section{Eosinophilic inflammation of airways in AIA}

Signs of persistent inflammation of the bronchi in patients with AIA are characterised by eosinophilic infiltration, desquamation of epithelia, increased cytokine production, and expression of adhesion molecules. ${ }^{7}$ Bronchial biopsy specimens reveal a fourfold increase in eosinophils in patients with AIA compared with aspirin tolerant asthmatic subjects. ${ }^{8}$ Mast cell counts are variable, probably reflecting activation of this cell population. Increased eosinophilia in the bronchi is accompanied by increased expression of interleukin (IL)-5, a cytokine directing maturation, activation, recruitment, and prevention of eosinophil apoptosis. Since the frequency of atopy is similar in patients with AIA and in aspirin tolerant asthmatics, ${ }^{9}$ persistent eosinophilic inflammation has to result from another mechanism. A chronic viral infection and/or autoimmunisation are likely events preceding the onset of AIA.$^{10}$ In fact, many patients with AIA have a low titre of anti-DNA antibodies, enhanced IgG synthesis, and associated HLA antigens.

Infiltrating eosinophils are a site of cysteinyl leukotriene (cys-LT) synthesis, as demonstrated by immunostaining of $\mathrm{LTC}_{4}$ synthase. The overexpression of $\mathrm{LTC}_{4}$ synthase in bronchial biopsy specimens of patients with AIA correlates with aspirin sensitivity and is several times higher than in aspirin tolerant asthmatic subjects. ${ }^{8}$

\section{Lipid mediators of asthma and aspirin sensitivity}

Despite numerous attempts, no immune system mediated hypersensitivity to aspirin has been found in patients with AIA. In addition, cross sensitivity to other non-steroidal antiinflammatory drugs (NSAIDs), represented by a variety of chemical compounds, would suggest that any immune response dependent mechanism is improbable. Cyclo-oxygenase (COX) is a common target for the action of NSAIDs. Careful comparison of inhibitory spectra of several NSAIDs suggests that inhibition of COX-1 rather than COX-2 triggers bronchoconstriction in sensitive patients. ${ }^{11}$ Measurements of COX metabolites in the body fluids, especially in bronchoalveolar lavage fluid, demonstrate diminished biosynthesis of prostaglandin $\mathrm{E}_{2}\left(\mathrm{PGE}_{2}\right)$ in patients with AIA. Administration of aspirin further suppresses $\mathrm{PGE}_{2}$ production, but this is not reflected by inhibition of the synthesis of other prostanoids as can be seen on COX inhibition in aspirin 


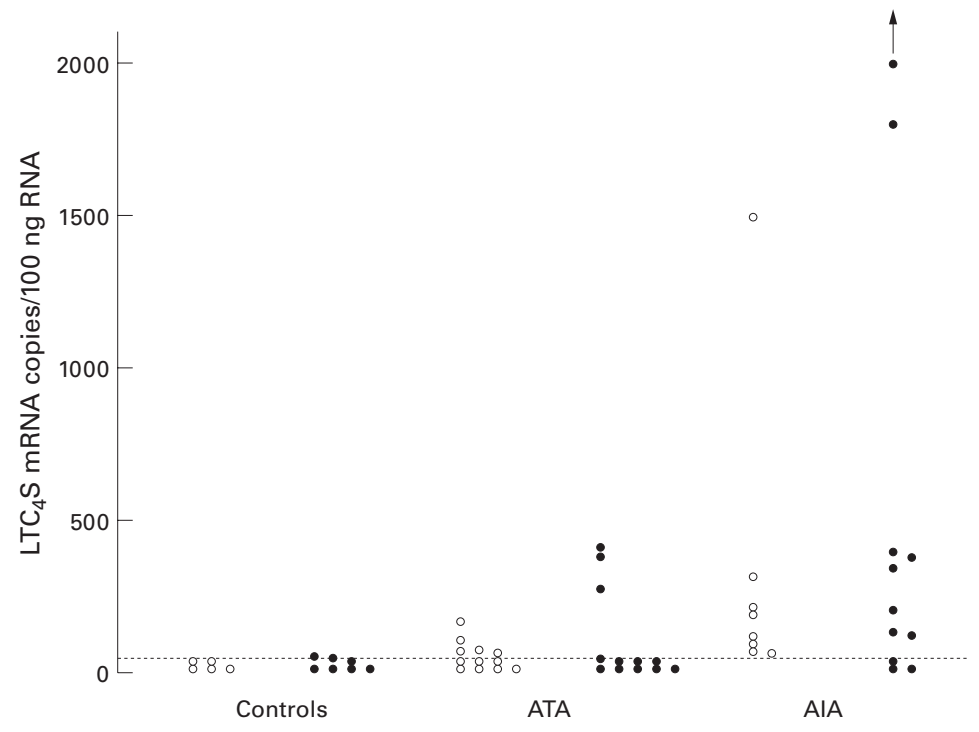

Figure $1 \mathrm{LTC}_{4}$ synthase (LTC $\mathrm{C}_{4}$ ) copies per 100ng total RNA calculated on the basis of semiquantitative competitive RT-PCT (sensitivity threshold 50 copies). Patients grouped according to their $L T C_{4} S$ genotypes. $\bullet=$ homozygotes $C C$ or heterozygotes $A C ; \circ=$ homozygotes $A A$. ATA = aspirin tolerant asthma; $A I A=$ aspirin induced asthma .

tolerant asthmatics. ${ }^{12} \mathrm{PGE}_{2}$ is a probable trigger for asthmatic attacks in AIA; inhibition of its synthesis removes $\mathrm{PGE}_{2}$ mediated restraints on inflammatory cells. This prostaglandin, in addition to its anti-inflammatory properties, can relax smooth muscles. Genetic screening of COX genes has not revealed any alterations to explain this phenomenon. $\mathrm{PGE}_{2}$ synthase, which is an inducible and glutathione dependent enzyme, has been newly cloned $^{13}$ but no studies in patients with AIA are yet available.

LTC $_{4}$ seems to be the most important bronchoconstrictor mediator of asthma in aspirin sensitive disease. ${ }^{14}$ Urinary excretion of $\mathrm{LTE}_{4}$ is increased by $2-10$ times in aspirin sensitive asthmatics compared with aspirin tolerant patients. During inhalatory aspirin challenge, a further transient increase in $\mathrm{LTE}_{4}$ excretion in the urine is observed in patients with AIA. ${ }^{15}$ Cys-LTs can be released into the nasal cavity and bronchi after local deposition of aspirin. ${ }^{12} 16$

The first step in the biosynthesis of cys-LTs is accomplished by 5-lipoxygenase (5-LO) and 5-LO associated protein (FLAP). Expression of 5-LO is modulated by a genetic variation in the SP1 transcription factor binding motif of the 5-LO gene, although no association between allelic variants of 5-LO gene promoter and AIA have been found. ${ }^{17}$ Immunostaining of bronchial biopsy specimens for 5-LO and FLAP have not revealed any difference with regard to aspirin sensitivity. ${ }^{8}$ Thus, only $\mathrm{LTC}_{4}$ synthase, a terminal enzyme for cys-LT pro-

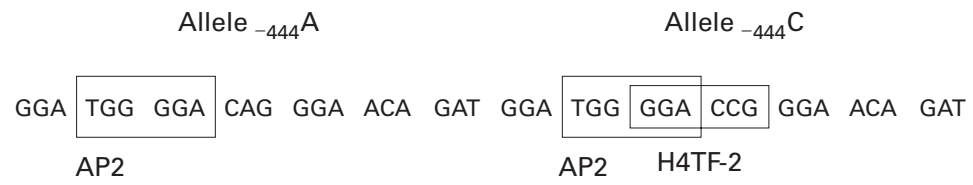

Figure 2 LTC $_{4}$ synthase ( $\left.L T C_{4} S\right)$ promoter sequence between nucleotides -454 and -434 upstream to the translation start. Allele $A$ has an AP2 transcription factor core sequence $(-451$ to -446$)$. Allele $C$ has the same AP2 binding motif which overlaps with a $H 4 T F-2$ binding ( -448 to -444$)$ site created by $-444 A \rightarrow C$ transversion. duction, is the pathway constituent distinguishing patients with AIA. Overexpression of $\mathrm{LTC}_{4}$ synthase could result from an intense enzyme induction in cells infiltrating bronchi of patients with AIA, but the possibility of a genetic mechanism facilitating the $\mathrm{LTC}_{4}$ synthase gene transcription has been tested.

Genetic polymorphism of $\mathrm{LTC}_{4}$ synthase controls biosynthesis of leukotrienes

LTC $_{4}$ synthase is expressed in eosinophils, basophils, and macrophages. In some other cells such as the epithelia, endothelia, and platelets the enzyme is an orphan because of lack of 5-LO expression. In these cells production of $\mathrm{LTC}_{4}$ synthase depends on external sources of $\mathrm{LTA}_{4}$. The $\mathrm{LTC}_{4}$ synthase gene has been cloned and its sequence, molecular organisation, and functional analysis have been described. ${ }^{18}$

A common allelic variant of the $\mathrm{LTC}_{4}$ synthase gene distinguished by the transversion of adenine (A) to cytosine (C), 444 bases upstream from the translation start, was found during screening of the $5^{\prime}$ region of the gene. ${ }^{19}$ In a population of asthmatic patients with more than 60 individuals in each group, the allele C frequency was $39 \%$ in patients with AIA but $26 \%$ in aspirin tolerant asthmatics and $25 \%$ in healthy controls. ${ }^{20}$ Semiquantitative studies of $\mathrm{LTC}_{4}$ synthase transcripts in peripheral blood eosinophils (fig 1 ) revealed increased numbers of mRNA copies in patients with AIA. This increase correlated with the presence of the allelic C variant of $\mathrm{LTC}_{4}$ synthase. ${ }^{21}$

By functional studies, vectors carrying either the $\mathrm{C}$ or A allele of the 5 ' end of the $\mathrm{LTC}_{4}$ synthase gene and expressed in eukaryotic cell lines showed that allele $\mathrm{C}$ produced a moderate $(25 \%)$ enhancement of the reporter gene transcription. ${ }^{22} \mathrm{~A}$ difference in binding of transcription factors created by the transversion has been analysed by oligonucleotide shift assay. Incubations of double strand oligonucleotides encompassing the transversion with nuclear extracts had a three times higher affinity of transcription factor AP2 for allele C. In addition, because of the overlap of the DNA motifs, allele $\mathrm{C}$ can interact with another transcription factor (fig 2), namely $\mathrm{H} 4 \mathrm{TF}-2$. An interaction of cytokine induced AP2 with a cell cycle dependent $\mathrm{H} 4 \mathrm{TF}-2$ is probably the cause of the higher expression of $\mathrm{LTC}_{4}$ synthase transcripts in circulating eosinophils of patients with AIA. The nature of the interaction which orchestrates $\mathrm{LTC}_{4}$ synthase expression in inflammatory cells of patients with AIA has not been fully elucidated. A transient increase in urinary $\mathrm{LTE}_{4}$ after aspirin challenge in sensitive patients (fig 3 ) is significant only in carriers of the C allele. ${ }^{23}$ Homozygotes for $\mathrm{LTC}_{4}$ synthase AA genotype are characterised by a lower dose of lysyl-aspirin (6.65 (SD 7.45) versus $30.0(47.0) \mathrm{mg}$ ) to provoke a fall in $\mathrm{FEV}_{1}$ of $20 \%\left(\mathrm{PD}_{20}\right)$. This negative correlation of aspirin $\mathrm{PD}_{20}$ with $\mathrm{LTC}_{4}$ synthase genotype $(\mathrm{p}<0.001)$ strongly suggests a complex mechanism for the disease, with a subpopulation of patients highly sensitive to aspirin as a result of a more efficient triggering mechanism. 


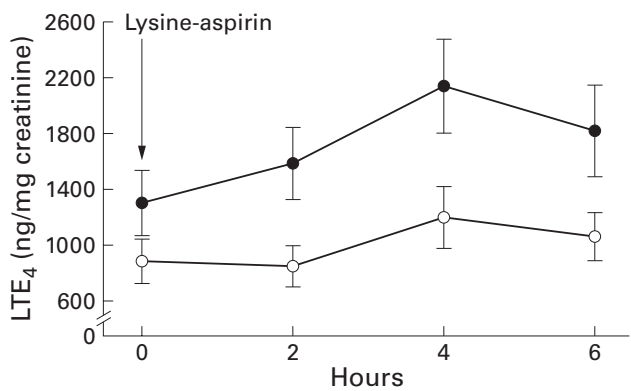

Figure 3 Urinary excretion of $L T E_{4}$ in $n g / m g$ creatinine in patients with AIA challenged with inhalatory aspirin $P_{20}$. Urine samples collected in two hour intervals. Patients were grouped according to their $\mathrm{LTC}_{4}$ synthase genotypes: - = homozygotes $C C$ or heterozygotes $A C ; \circ=$ homozygotes AA. Error bars = standard error of mean.

\section{Conclusions}

Aspirin sensitive asthmatic subjects have overexpression of $\mathrm{LTC}_{4}$ synthase in their airways. Increased production of cys-LTs at baseline and in response to aspirin provocation suggests that these mediators are the main cause of the disease. Genetic polymorphism of the promoter region of the $\mathrm{LTC}_{4}$ synthase gene effects its expression in cells by an increase in the transcription rate in eosinophils and a change in its inducibility in response to proinflammatory mediators. The association of allelic variant $\mathrm{C}$ of $\mathrm{LTC}_{4}$ synthase with AIA is moderate and similar to other genetic polymorphisms contributing to common diseases. Aspirin induced asthma is a disease manifested by dysregulation of eicosanoid synthesis after exposure to NSAIDs which is enhanced by the genetically determined overexpression of $\mathrm{LTC}_{4}$ synthase.

1 Szczeklik A. Aspirin-induced asthma. In: Vane JR, Botting RM, eds. Aspirin and other salicylates. London: Chapman \& RM, eds. Aspirin and other salicy

2 Online Mendelian Inheritance in Men, OMIM, Johns Hopkins University, Baltimore, Md. MIM Number: 208550 : kins University, Baltimore, Md. MIM Number: 208550:
1998. World Wide Web URL: http:// 1998. World Wwincbi.nlm.nih.gov/omim/

3 Miller FF. Aspirin-induced bronchial asthma in sisters. Ann Allergy 1971;29:263-5.

4 Lockey RF, Rucknagel DL, Vanselow NA. Familial occurrence of asthma, nasal polyps and aspirin intolerance. Ann Intern Med 1973;78:57-63.
5 von Maur K, Adkinson Jr NF, Van Merter Jr TE, et al. Aspirin intolerance in a family. F Allergy Clin Immunol 1974;54: 380-95.

6 Nizankowska E, Duplaga M, Bochenek G, et al. Clinical course of aspirin-induced asthma: results of AIANE. In: Szczeklik A, Gryglewski RJ, Vane RJ, eds. Eicosanoids, aspirin and asthma. New York: Marcel Dekker, 1998: 451-72.

7 Nasser SMS, Pfister R, Christie PE, et al. Inflammatory cell populations in bronchial biopsies from aspirin-sensitive asthmatic subjects. Am F Respir Crit Care Med 1996;153: 90-6.

8 Cowburn AS, Sladek K, Soja J, et al. Overexpression of leukotriene $\mathrm{C} 4$ synthase in bronchial biopsies from patients with aspirin-intolerant asthma. F Clin Invest 1998;101:113.

9 Bochenek G, Nizankowska E, Szczeklik A. Atopy trait in hypersensitivity to nonsteroidal anti-inflammatory drugs. Allergy 1996;51:16-23

10 Szczeklik A. Aspirin-induced asthma as a viral disease. Clin Allergy 1988;18:15-20.

11 Szczeklik A, Stevenson DD. Aspirin-induced asthma: advances in pathogenesis and management. F Allergy Clin Immunol 1999;104:5-13.

12 Szczeklik A, Sladek K, Dworski R, et al. Bronchial aspirin challenge causes specific eicosanoid response in aspirin sensitive asthmatics. Am F Respir Crit Care Med 1996;154: 1608-14

13 Jakobsson PJ, Thoren S, Morgenstern R, et al. Identification of human prostaglandin E synthase: a microsomal, glutathione-dependent, inducible enzyme, constituting a potential novel drug target. Proc Natl Acad Sci USA 1999; 96:7220-5.

14 Israel E, Fisher AR, Rosenberg MA, et al. The pivotal role of 5-lipoxygenase products in the reaction of aspirin-sensitive
asthmatics to aspirin. Am Rev Respir Dis 1993;148:144751 .

15 Christie PE, Tagari P, Hutchinson AW, et al. Urinary leukotriene $\mathrm{E} 4$ concentrations increase after aspirin challenge in aspirin-sensitive asthmatic subjects. Am Rev Respir Dis 991;143:1025-9.

16 Kowalski ML, Sliwinska-Kowalska M, Igarashi Y, et al. Nasal sercretions in response to acetylsalicylic acid. $\mathcal{f}$ Allergy Clin Immunol 1993;91:580-98.

17 In KH, Asano K, Beier D, et al. Naturally occurring mutations in the human 5-lipoxygenase gene promoter that modify transcription factor binding and reporter gene transcription. $\mathcal{F}$ Clin Invest 1997; 99:1130-7.

18 Lam BK, Penrose JF, Freeman GJ, et al. Expression cloning of a cDNA for human leukotriene C4 synthase, an integral membrane protein conjugating reduced glutathione to leukotriene A4. Proc Natl Acad Sci USA 1994;91:7663-7.

19 Sanak M, Simon H-U, Szczeklik A Leukotriene C4 Sanak M, Simon H-U, Szczeklik A. Leukotriene C4
synthase promoter polymorphism and risk of aspirininduced asthma. Lancet 1997;350:1599-600.

20 Sanak M, Szczeklik A. Leukotriene C4 synthase promoter polymorphism in bronchial asthma. Allergy 1998; 53:1123S

21 Sanak M, Bazan-Socha S, Szczeklik A. Leukotriene C4 synthase (LTC4S) expression in blood eosinophils of patients with asthma. Eur Respir 7 1998;12:373S.

22 Sanak M, Bazan-Socha S, Pierzchalska M, et al. Upregulation of peptidoleukotrienes pathway in aspirin-intolerant to a constitutive activation of altered asthmatics is related to a constitutive activation of altered
leukotriene C4 synthase promoter. Am 7 Resp Crit Care Med 1999; 159:A650.

23 Szczeklik A, Sanak M, Nizankowska E, et al. Leukotriene C4 synthase genetic polymorphism directs urinary cysteinylleukotriene response to aspirin challenge in asthma. Allergy 1998;53:61S. 